\title{
Long non-coding RNA EWSAT1 contributes to the proliferation and invasion of glioma by sponging miR-152-3p
}

\author{
HUI YANG $^{1 *}$, WEIDA CHEN ${ }^{2 *}$, GUANGYU JIANG ${ }^{3}$, \\ JUN YANG ${ }^{3}$, WEIFENG WANG ${ }^{3}$ and HONGBIN $\mathrm{LI}^{3}$ \\ Departments of ${ }^{1}$ Neurology, ${ }^{2}$ Cardiology and ${ }^{3}$ Neurosurgery, The First Affiliated Hospital of Jiamusi University, \\ Jiamusi, Heilongjiang 154001, P.R. China
}

Received November 20, 2019; Accepted April 17, 2020

DOI: $10.3892 / \mathrm{ol} .2020 .11716$

\begin{abstract}
Long non-coding RNAs (IncRNA) are a type of ncRNA with a length ranging from 200-1,000 nucleotides. Previous studies have confirmed that the lncRNA Ewing sarcoma associated transcript 1 (EWSAT1) exerts regulatory roles in cancer development and progression. However, its clinical significance in glioma remains unknown. In the present study, RNA-sequencing data from the Gene Expression Omnibus database and The Cancer Genome Atlas was explored to investigate the association between EWSAT1 expression and prognosis in patients with glioma. Increased EWSAT1 was associated with the presence of necrosis on magnetic resonance imaging scans in patients with glioma. Furthermore, knockdown of EWSAT1 was indicated to suppress the proliferative and invasive abilities of glioblastoma cell lines using Cell Counting Kit-8 and Transwell assays. Additionally, microRNA (miR)-152-3p was identified as a potential target of EWSAT1. The present study demonstrated that EWSAT1 interacted directly with miR-152-3p, and rescue experiments confirmed that EWSAT1 participated in glioma development by suppressing miR-152-3p. These results indicated that EWSAT1 is involved in the occurrence and progression of glioma, and may serve as a novel target and potential prognostic biomarker of glioma treatment.
\end{abstract}

\section{Introduction}

Glioblastoma (GBM) is a malignant and aggressive primary nervous system tumors worldwide, with a high rate of

Correspondence to: Professor Hongbin Li, Department of Neurosurgery, The First Affiliated Hospital of Jiamusi University, 348 Dexiang Road, Xiangyang, Jiamusi, Heilongjiang 154001, P.R. China

E-mail: lihbneuro@126.com

*Contributed equally

Key words: glioblastoma, Ewing sarcoma associated transcript 1, microRNA-152-3p, invasion recurrence and a poor prognosis compared with other types of nervous system tumor (1). As reported in the 2016 World Health Organization classification, there are four different grades, and two classes, of glioma: Grades I and II (low-grade gliomas); and grades III and IV (high-grade gliomas) (2). Despite the advances in molecular biology and oncogenetics for the treatment of patients with glioma over the previous decades, the prognosis and overall survival (OS) time, measured as $12-14$ months following surgical resection, remain poor $(3,4)$. However, the molecular mechanisms of glioma tumorigenesis remain unclear (5-8). Therefore, it is essential to identify novel therapeutic and prognostic targets in glioma.

Long non-coding RNAs (lncRNAs) are a class of newly identified RNAs with a length ranging from 200 to 1,000 nucleotides, which lack protein-coding ability $(9,10)$. lncRNAs regulate the expression of genes at epigenetic, transcriptional and post-transcriptional levels. Previous evidence has indicated that the abnormal expression of lncRNAs can affect glioma development and tumorigenesis (11). These lncRNAs may serve as useful molecular targets for the diagnosis of malignant tumors, including $\operatorname{GBM}(11,12)$. The biological role of lncRNAs in glioma remains to be investigated.

lncRNA Ewing sarcoma associated transcript 1 (EWSAT1) is a novel cancer-associated lncRNA that serves critical roles in the occurrence and progression of tumors, including nasopharyngeal carcinoma $(13,14)$, osteosarcoma $(15)$, colorectal $(16)$ and ovarian cancer (17), and Ewing sarcoma (18). However, the biological role and mechanism of EWSAT1 in gliomas remain to be identified.

Previous studies on the function of microRNA (miRNA/miR) have demonstrated that miRNAs are involved in the regulation of gene expression, cell differentiation, metabolism and invasion $(19,20)$. Previous studies have indicated that miR-152-3p is closely associated with carcinogenesis, including glioma (21-23). Therefore, the present study aimed to explore the potential association between EWSAT1 and $\mathrm{miR}-152-3 \mathrm{p}$ in glioma.

The present study investigated the expression and molecular mechanism of EWSAT 1 in glioma. The results suggested that EWSAT1 was increased in glioma. In addition, EWSAT1 knockdown suppressed the proliferative and invasive abilities of glioma cells. The primary aim of the present study was to 
investigate the function of lncRNA EWSAT1, and to detect the role of miRNA-152-3p in the regulatory mechanism of glioma via IncRNA EWSAT1. The results may provide a theoretical basis for developing new therapeutic drugs against glioma.

\section{Materials and methods}

Cell lines and clinical tissues. A total of 5 glioma cell lines (U251, T98G, LN229, A172 and SHG44) were obtained from The Cell Bank of Type Culture Collection of the Chinese Academy of Sciences. Normal human astrocyte (NHA) cells were purchased from The Institute of Biochemistry and Cell Biology, Chinese Academy of Sciences. All cells were sub-cultured in Dulbecco's modified Eagle's medium (DMEM; Gibco; Thermo Fisher Scientific, Inc.) supplemented with $10 \%$ fetal bovine serum (FBS; Gibco; Thermo Fisher Scientific, Inc.) and maintained in a humidified incubator at $37^{\circ} \mathrm{C}$ with $5 \% \mathrm{CO}_{2}$.

Human GBM samples and adjacent normal tissues were collected via surgical resection performed at The First Affiliated Hospital of Jiamusi University. Samples were collected from patients with GBM (27 females and 15 males; age range, 31-72 years; median, 51.34 years) between January 2011 and December 2017. None of the patients received any therapy prior to surgery. All GBM samples were confirmed by two senior pathologists. The study protocol was approved by the Ethics Committee of The Institutional Review Board of Jiamusi University (approval no. JUIRBR-2019-214), and all procedures were performed in accordance with the principles outlined in The Declaration of Helsinki (24). Written informed consent was provided by all patients to participate in the study. The clinical characteristics of the patients are summarized in Table I.

Bioinformatic prediction of EWSAT1 expression. The edgeR software package (Bioconductor) in RStudio 3.5.1 (https://www. rstudio.com/; RStudio, Inc.) was used to detect the differentially expressed lncRNAs in normalized gene expression profile data from the Gene Expression Omnibus (GEO) database (https://www.ncbi.nlm.nih.gov/gds/?term=GSE4290) and The Cancer Genome Atlas (TGCA) GBM database (http://cancergenome.nih.gov) $(25,26)$. For the normalized gene expression profile data, the edgeR package was employed to explore significantly abnormally expressed IncRNAs. Log2 fold-change (FC) $>2$ and false-discovery rate (FDR) $<0.01$ were selected as significantly cut-off values, and the aberrantly expressed candidate lncRNAs were identified. Clinical prognosis data were acquired from the Gene Expression Profiling Interactive Analysis (GEPIA) database (http://gepia.cancer-pku.cn/). Gene Ontology (GO) term enrichment analysis was examined using the Database for Annotation, Visualization and Integrated Discovery version 6.8 (https://david.ncifcrf.gov/).

Reverse transcription-quantitative PCR (RT-qPCR). TRIzol ${ }^{\circledR}$ and chloroform (Invitrogen; Thermo Fisher Scientific, Inc.) were utilized to extract total cellular RNA from each group, according to the manufacturer's protocol. cDNA was synthesized using the PrimeScript ${ }^{\mathrm{TM}}$ RT kit (Takara Biotechnology Co., Ltd.), according to the manufacturer's protocol. qPCR was performed according to the manufacturer's instructions of the TransScript Green Two-Step RT-qPCR SuperMix kit (Beijing Transgen Biotech Co., Ltd.). Thermocycling conditions consisted of an initial denaturing step at $94^{\circ} \mathrm{C}$ for $10 \mathrm{~min}$, followed by 40 cycles of denaturing at $94^{\circ} \mathrm{C}$ for $5 \mathrm{sec}$, annealing at $60^{\circ} \mathrm{C}$ for $30 \mathrm{sec}$ and extending at $72^{\circ} \mathrm{C}$ for $45 \mathrm{sec}$. The primers used were as follows: EWSAT1 forward, $5^{\prime}-\mathrm{GTG}$ TCTGGCAAGGAACACTA-3' and reverse, 5'-GGTGGA GAAGAGGGACAAT-3'; miR-152-3p forward, 5'-GCGCTC AGTGCATGACAGA-3' and reverse, 5'-GTCGTATCCAGT GCAGGGT-3'; matrix metalloproteinase (MMP)-2 forward, 5'-CAGGACATTGTCTTTGATGG-3' and reverse, 5'-TGA AGAAGTAGCTATGACCA-3'; MMP-9 forward, 5'-AGA CCTGGGCAGATTCCAAAC-3' and reverse 5'-CGGCAA GTCTTCCGAGTAGT-3'; U6 forward, 5'-GGATATTGTTGC CATCAATGACC-3' and reverse, 5'-AGCCTTCTCCATGGT GGTGAAGA-3'; and GAPDH forward 5'-AAGAAGGTG GTGAAGCAGGC-3' and reverse, 5'-GTCAAAGGTGGA GGAGTGGG-3'. U6 served as the endogenous control. The results were calculated using the $2^{-\Delta \Delta \mathrm{Cq}}$ method (27).

Plasmid transfection. The U251 and T98G cells were maintained in DMEM containing $10 \% \mathrm{FBS}$ at $37^{\circ} \mathrm{C}$ in $5 \% \mathrm{CO}_{2}$. When the cell confluence reached $\sim 80 \%$, the cells were transfected with $20 \mu \mathrm{M}$ of each construct (siRNA or miRNA mimics) using Lipofectamine ${ }^{\circledR} 2000$ (Invitrogen; Thermo Fisher Scientific, Inc.), according to the manufacturer's protocol and incubated at $37^{\circ} \mathrm{C}$ for $6 \mathrm{~h}$. The culture medium was subsequently replaced with fresh DMEM supplemented with $10 \%$ FBS, and subsequent experimentation was performed $24 \mathrm{~h}$ post-transfection. Small interfering RNA (siRNA) targeting EWSAT1 and its negative control (si-NC), miR-152-3p mimic or inhibitor, and their respective negative control (miR-NC) $(20 \mu \mathrm{M})$ were obtained from Shanghai GenePharma Co., Ltd. The sequences were as follows: EWSAT1 siRNA forward, 5'-UUGGGCUCUCAAUGGUAUCAU-3' and reverse, 5'-AAG GGAGGGUUACUAACUUUA-3'; si-NC forward, 5'-GGU AAGCAGUGGCUCCUCUAA-3' and reverse, 5'-ACGUGA CACGUUCGGAGAAUU-3'; miR-152-3p mimics forward, 5'-UCAGUGCAACUGACAGAACUUGG-3' and reverse, 5'-UAGCCACGGUUGUGUAAAGUCUG-3'; miR-152-3p inhibitor forward, 5'-CGCGCUAGCAGCACGUAAAU-3' and reverse, 5'-GUGCAGGGUCCGAGGUCAUC-3'; and miR-NC forward, 5'-CAGUACUUUUGUGUAGUACAA-3' and reverse, 5'-CAGUACUUUUGUGUAGUACAA-3'. Transfection with Lipofectamine ${ }^{\circledR} 2000$ (Invitrogen; Thermo Fisher Scientific, Inc.) was performed according to the manufacturer's protocol. The cells were then cultured for subsequent experiments and obtained at $24 \mathrm{~h}$ after transfection.

Dual-luciferase reporter assay. To detect the potential target miRNAs of EWSAT1, the TargetScan (www.targetscan. org/) and StarBase (http://starbase.sysu.edu.cn/) databases were explored. Among all the statistically relevant miRNAs, miR-152-3p exhibited the highest score in the two databases and was selected for further experiments. The wild-type (WT) or mutant (MUT) putative miR-152-3p binding sites of the 3'-untranslated region (UTR) in EWSAT1 were ligated into the pMIR-luciferase reporter plasmid vector (Shanghai GenePharma Co., Ltd.). U251 cells were added in 6-well plates at $1 \times 10^{5}$ cells/well and the recombinant vectors were co-transfected with miR-152-3p mimics or miR-NC using Lipofectamine ${ }^{\circledR} 2000$. The medium was replaced at $6 \mathrm{~h}$ 
Table I. Clinical characteristics of the patients with glioblastoma according to long non-coding RNA EWSAT1 level in tissues $(\mathrm{n}=42)$.

EWSAT1 expression

\begin{tabular}{|c|c|c|c|c|}
\hline \multirow{2}{*}{ Variables } & \multirow[b]{2}{*}{$\mathrm{N}$} & & \multirow[b]{2}{*}{ P-value } \\
\hline & & Low & High & \\
\hline \multicolumn{5}{|l|}{ Age, years } \\
\hline$<60$ & 23 & 9 & 14 & \multirow[t]{2}{*}{0.382} \\
\hline$\geq 60$ & 19 & 10 & 9 & \\
\hline \multicolumn{5}{|l|}{ Sex } \\
\hline Male & 15 & 9 & 6 & \multirow[t]{2}{*}{0.152} \\
\hline Female & 27 & 10 & 17 & \\
\hline \multicolumn{5}{|c|}{ Karnofsky Performance Scale score } \\
\hline$<60$ & 24 & 11 & 13 & \multirow[t]{2}{*}{0.929} \\
\hline$\geq 60$ & 18 & 8 & 10 & \\
\hline \multicolumn{5}{|c|}{ Mean tumor diameter, $\mathrm{cm}$} \\
\hline$<5$ & 29 & 12 & 17 & \multirow[t]{2}{*}{0.453} \\
\hline$\geq 5$ & 13 & 7 & 6 & \\
\hline \multicolumn{5}{|c|}{ Necrosis on MRI } \\
\hline Yes & 27 & 9 & 18 & \multirow[t]{2}{*}{$0.038^{\mathrm{a}}$} \\
\hline No & 15 & 10 & 5 & \\
\hline \multicolumn{5}{|l|}{ Seizure } \\
\hline Yes & 14 & 5 & 9 & \multirow[t]{2}{*}{0.381} \\
\hline No & 28 & 14 & 14 & \\
\hline
\end{tabular}

${ }^{\mathrm{a} P}<0.05$. EWSAT1, Ewing sarcoma associated transcript 1; MRI, magnetic resonance imaging.

post-transfection, and the firefly and Renilla luciferase signals were examined $48 \mathrm{~h}$ following transfection using the Dual Luciferase Reporter Assay kit (Promega Corporation). Firefly luciferase activity was normalized to that of Renilla luciferase.

Cell proliferation assays. U251 and T98G cell proliferation was performed by Cell Counting Kit-8 assay (CCK-8; Beyotime Institute of Biotechnology) at 24, 48 and $72 \mathrm{~h}$ according to the manufacturer's protocol. The transfected glioma cells were added to 96-well plates at a density of 3,000 cells/well. Then, a culture solution containing $10 \mu \mathrm{l}$ CCK-8 regent was added. Following incubation at $37^{\circ} \mathrm{C}$ for additional $4 \mathrm{~h}$, cell viability was measured by detecting the absorbance at a wavelength of $490 \mathrm{~nm}$ using a microplate reader (Bio-Rad Laboratories, Inc.)

Transwell invasion assays. The upper chambers of Transwell plates were precoated with Matrigel ${ }^{\boxplus}$ (Corning Life Sciences) at $37^{\circ} \mathrm{C}$ for $30 \mathrm{~min}$. Subsequently, U251 and T98G cells were suspended with serum-free DMEM $\left(5 \times 10^{4}\right)$ and seeded in the upper chambers. DMEM supplemented with $20 \%$ FBS was added to the lower chambers and the plates were incubated in $37^{\circ} \mathrm{C}$ with $5 \% \mathrm{CO}_{2}$ for $24 \mathrm{~h}$. Cells were fixed with $4 \%$ paraformaldehyde at room temperature for $10 \mathrm{~min}$, and stained with hematoxylin and eosin (5 min for hematoxylin and $1 \mathrm{~min}$ for eosin at room temperature), using the Staining kit (Beijing Solarbio Science \& Technology Co., Ltd.). The number of migratory cells per sample was counted in 5 randomly selected fields under a light microscope (Nikon Corporation) at magnification, $\mathrm{x} 100$.

Western blotting. Proteins were extracted from cell lines (U251 and T98G) with lysis buffer (BIOSS). The quantity of protein was detected using the BCA Protein Assay kit (Beyotime Institute of Biotechnology), and the lysates (20 $\mu \mathrm{g}$ protein) were separated on 10\% SDS-PAGE and electrophoretically transferred onto PVDF membranes (Beyotime Institute of Biotechnology). Following blocking in 5\% skim milk for $1 \mathrm{~h}$ at $37^{\circ} \mathrm{C}$ and incubation with the corresponding primary antibodies at $4^{\circ} \mathrm{C}$ overnight [anti-MMP-2 (rabbit polyclonal antibody; 1:1,000; cat. no. 40094s; Cell Signaling Technology, Inc.), anti-MMP-9 (rabbit polyclonal antibody; 1:1,000; cat. no. 13667; Cell Signaling Technology, Inc.) and anti-GAPDH (mouse monoclonal antibody; 1:1,000; SC-47724; Santa Cruz Biotechnology, Inc.)], the membranes were incubated with horseradish peroxidase-conjugated anti-mouse or anti-rabbit secondary antibody (cat. no. ab6721 and ab6728; 1:2,000; Abcam) for $1 \mathrm{~h}$ at room temperature. Then, the bands were visualized using ECL (Beyotime Institute of Biotechnology) and analyzed with a ChemiDoc ${ }^{\mathrm{TM}}$ MP Imaging detection system (Bio-Rad Laboratories, Inc.) and Image Lab software 3.0 (Bio-Rad Laboratories, Inc.).

Immunofluorescence staining. U251 cells $\left(1 \times 10^{5}\right)$ were collected, and cell slides were prepared. Glass slides were stained with $0.1 \%$ poly-L-lysine at $4{ }^{\circ} \mathrm{C}$ overnight. Then, 

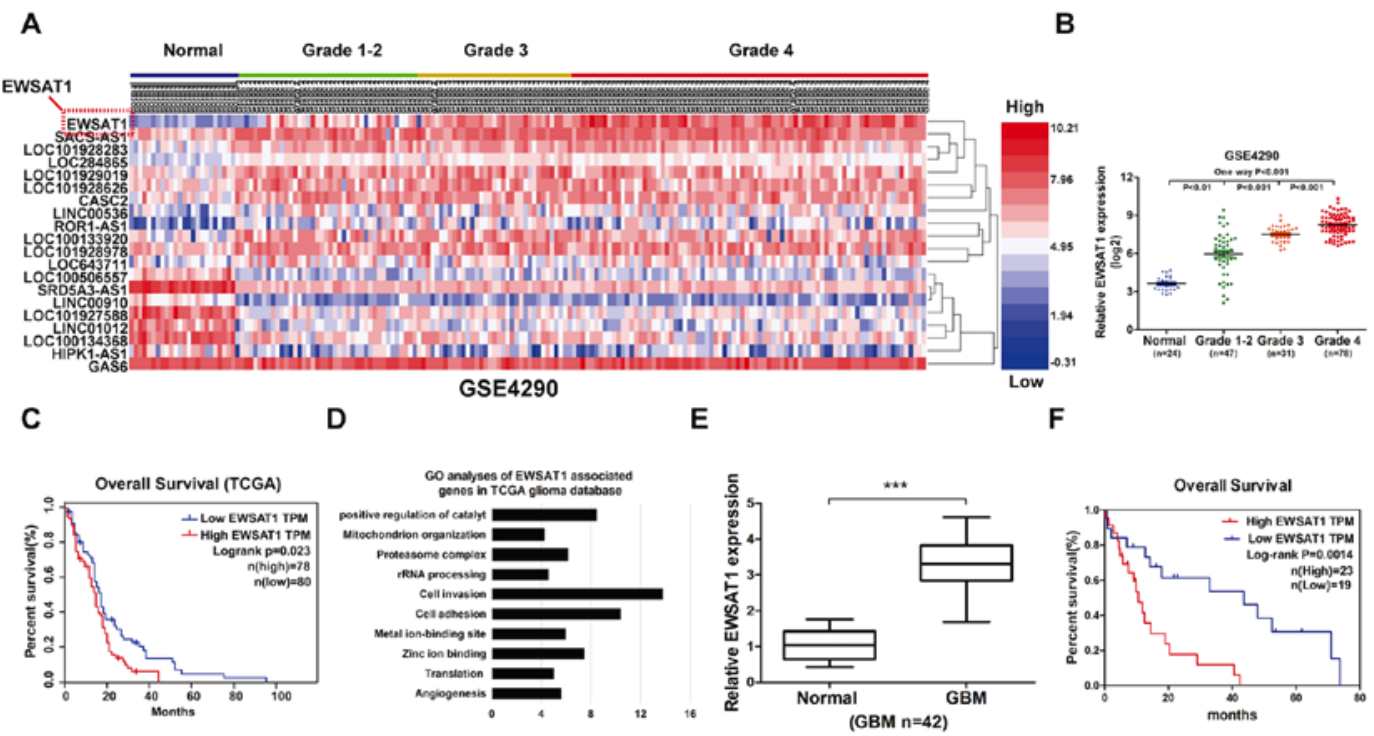

Figure 1. IncRNA EWSAT1 is upregulated in human GBM samples and is correlated with worse survival. (A) A heatmap demonstrated the aberrant expression of lncRNAs from the GEO database. Red bar represents high expression, while blue bar represents low expression. (B) Gene expression levels of EWSAT1 in the GEO cohort compared with the normal cohort. (C) Kaplan-Meier curve of overall survival in the GEO database, demonstrating that a high level of EWSAT1 is associated with poor prognosis $(n=158)$. (D) Gene function of EWSAT1-associated genes were identified using GO analysis. (E) An increased gene expression level of EWSAT1 was present in clinical tissues of patients with GBM $(n=42)$ compared with matched adjacent normal tissue, as determined by reverse transcription-quantitative PCR analysis. (F) Kaplan-Meier curve of overall survival in patients with glioma indicated that a high level of EWSAT1 was correlated with worse prognosis $(\mathrm{n}=42)$. Each experiment was performed in triplicate. ${ }^{* * * *} \mathrm{P}<0.001$. 1ncRNA, long non-coding RNA; EWSAT1, Ewing sarcoma associated transcript 1; GBM, glioblastoma; GO, Gene Ontology; TCGA, The Cancer Genome Atlas; GEO, Gene Expression Omnibus; TPM, Trans Per Kilobase of exon model per Million.

4\% paraformaldehyde with 5\% BSA (cat. no. 9048-46-8; Sigma Aldrich; Merck KGaA) was added for $20 \mathrm{~min}$, and the slides were fixed with $0.1 \%$ Triton $\mathrm{X}-100$ for $10 \mathrm{~min}$ at room temperature. The slides were then incubated with rabbit polyclonal primary antibodies against MMP-2 (cat. no. 40094s; 1:1,000; Cell Signaling Technology, Inc.) and MMP-9 (cat. no. 13667; 1:1,000; Cell Signaling Technology, Inc.) at $4^{\circ} \mathrm{C}$ for $1 \mathrm{~h}$, followed by incubation with the corresponding fluorescence-labeled rabbit secondary antibodies [tetramethylrhodamine (TRITC)-conjugated goat anti-rabbit IgG (cat. no. SA00007-2; 1:100; ProteinTech Group, Inc.) and fluorescein isothiocyanate (FITC)-conjugated goat anti-rabbit IgG (cat. no. SA00003-2; ProteinTech Group, Inc.)] at room temperature for $1 \mathrm{~h}$. Cell nuclei were then incubated with DAPI at $4^{\circ} \mathrm{C}(1 \mu \mathrm{g} / \mathrm{ml}$; cat. no. $4083 \mathrm{~s}$; Cell Signaling Technology, Inc.) for $15 \mathrm{~min}$ and observed under a fluorescence microscope (Nikon Corporation) at magnification, $\mathrm{x} 400$.

Statistical analysis. Data were expressed as the mean \pm standard deviation of 3 independent experiments. SPSS 21.0 (IBM Corp.) was applied for data analysis excluding the Kaplan-Meier. Student's t-test or one-way ANOVA with Tukey's post hoc test were performed to determine differences between groups. The associations between EWSAT1 level and the clinicopathological characteristics of the patients were analyzed using the $\chi^{2}$ or Fisher's exact tests. Pearson's correlation analysis was performed to determine the correlation between EWSAT1 and miR-152-3p expression levels. GraphPad Prism software 5.0 (GraphPad Software, Inc.) was used to detect the Kaplan-Meier curve and a log-rank test was performed to assess the survival percentage. The aberrantly expressed lncRNAs were assessed based on the Benjamini-Hochberg method (28). $\mathrm{P}<0.05$ was considered to indicate a statistically significant difference.

\section{Results}

Upregulation of EWSAT1 in GBM. Using the GEO dataset GSE4290, the top 20 aberrantly expressed lncRNAs were identified with cut-off values of $\log 2 \mathrm{FC}>2$ and FDR $<0.01$ (Fig. 1A). EWSAT1 was the most significantly differentially expressed lncRNA. EWSAT1 was identified to be upregulated in glioma tissues, and the level increased according to glioma grade (Fig. 1A and B). The clinical survival data collected from the GEPIA database (http://gepia.cancer-pku.cn/) demonstrated that increased EWSAT1 levels in the GBM samples were associated with a poorer OS, according to the median survival time of patients $(\mathrm{P}=0.023 ; \mathrm{n}=158$; Fig. $1 \mathrm{C})$. To detect the potential role of EWSAT1 in glioma progression, the associated gene expression profiles were explored by using the (GO) database (https://david.ncifcrf.gov/). It was observed that the most significant GO biological processes mainly included cell invasion and adhesion (Fig. 1D). GBM is the most malignant and aggressive primary nervous system tumor worldwide $(1,2)$, thus it is essential to detect the clinical characteristics of patients with GBM (Grade IV in glioma), according to EWSAT1 expression in tissues (GBM, $n=42)$. In the clinical GBM samples, the results of the present study demonstrated that the expression level of EWSAT1 exhibited a significant association with patient necrosis on magnetic resonance imaging scans (grade IV; Table I; $\mathrm{P}=0.038$ ). In addition, a higher level of EWSAT1 was observed in patients with GBM (grade IV) compared with that observed in adjacent normal brain tissues (Fig. 1E). The 
A

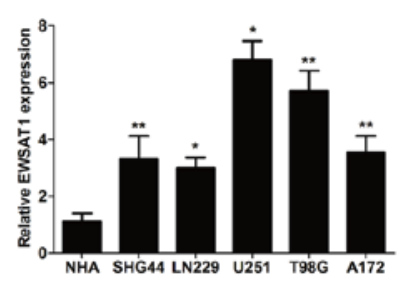

B

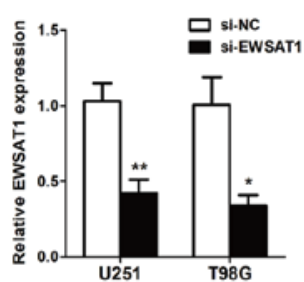

C
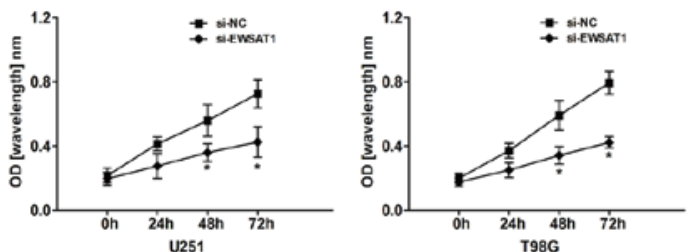

U251

D
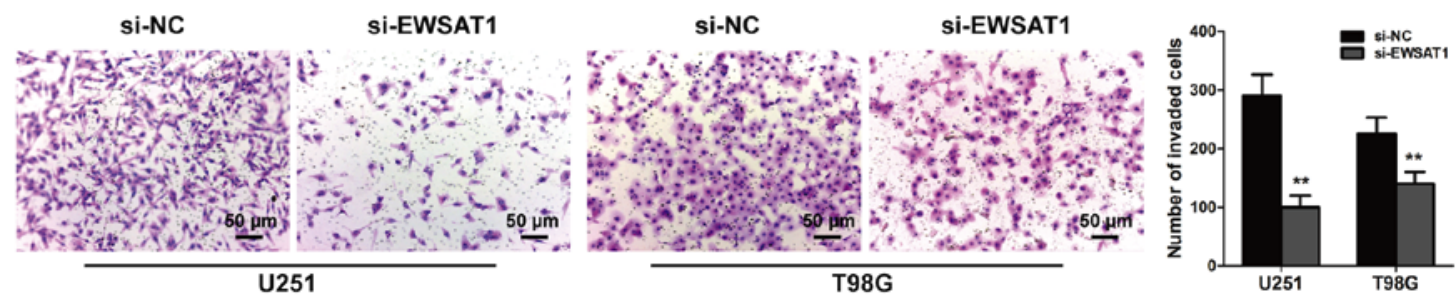

Figure 2. Silencing of the long non-coding RNA EWSAT1 inhibits the proliferative and invasive abilities of glioblastoma cell lines in vitro. (A) The EWSAT1 gene expression levels in the GBM U251, LN229, T98G, A172 and SHG44 cell lines compared with those in normal human astrocyte cells were assessed by RT-qPCR analysis. (B) EWSAT1 gene expression was efficiently knocked down by siRNA in U251 and T98G cells compared with the si-NC group, as detected by RT-qPCR assay. (C) GBM cell growth was measured using the Cell Counting Kit-8 assay (si-EWSAT1 vs. si-NC). (D) Transwell invasion assay was used to detect the invasive ability. Data are presented as the mean \pm standard deviation of 3 independent experiments. Magnification, $\mathrm{x} 100$; scale bar, $50 \mu \mathrm{m}$. ${ }^{*} \mathrm{P}<0.05$ and ${ }^{* *} \mathrm{P}<0.01$. EWSAT1, Ewing sarcoma associated transcript 1; GBM, glioblastoma; RT-qPCR, reverse transcription-quantitative PCR; NC, negative control; siRNA, small interfering RNA.

A

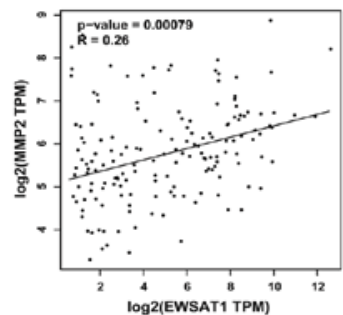

C
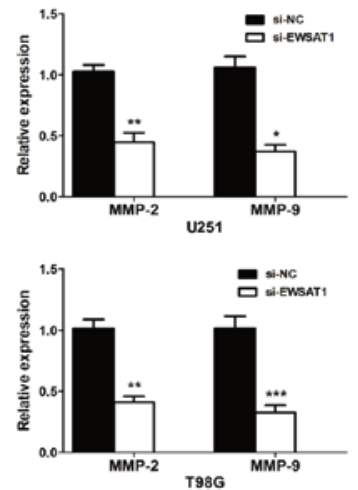

B

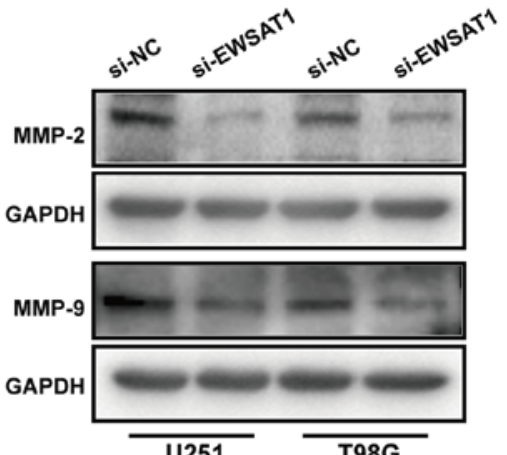

D

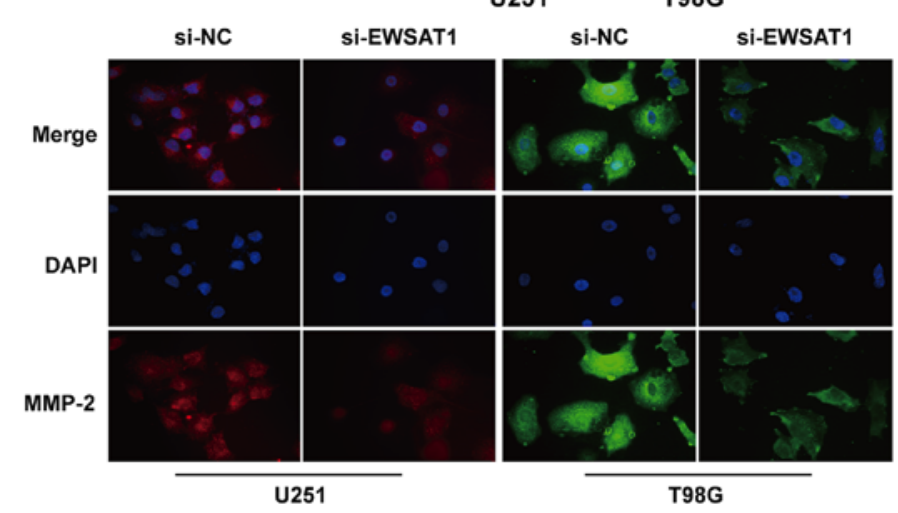

Figure 3. Expression of invasion-associated markers in GBM cells. (A) Correlation between long non-coding RNA EWSAT1 and MMP-2/MMP-9 expression in The Cancer Genome Atlas GBM database. (B and C) Following transfection with si-EWSAT1 or si-NC, the protein and mRNA expression levels of invasion-associated biomarkers MMP-2 and MMP-9 were demonstrated. (D) Immunofluorescence staining assay demonstrated a decreased level of MMP-2 in si-EWSAT1-transfected cells. Magnification, $\mathrm{x} 400 .{ }^{*} \mathrm{P}<0.05,{ }^{* *} \mathrm{P}<0.01$ and ${ }^{* * * *} \mathrm{P}<0.001$. The experiments were repeated 3 times. GBM, glioblastoma; EWSAT1, Ewing sarcoma associated transcript 1; MMP, matrix metalloproteinase; siRNA, small interfering RNA; NC, negative control.

Kaplan-Meier curves also exhibited a worse prognosis in patients with glioma expressing a high level of plasmacytoma variant translocation $1(\mathrm{P}=0.0014$; Fig. $1 \mathrm{~F})$. Thus, EWSAT1 was identified to be involved in the progression of glioma.
Knockdown of EWSAT1 suppresses GBM cell viability and invasion. As aforementioned, upregulated EWSAT1 levels were demonstrated in glioma cells. Therefore, it was hypothesized that EWSAT1 serves as a regulator of glioma 
A

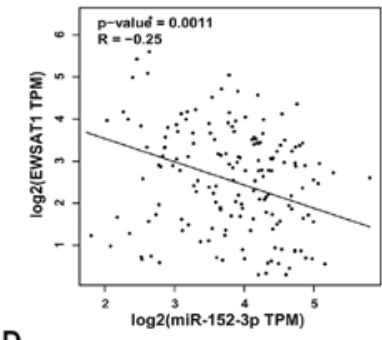

D

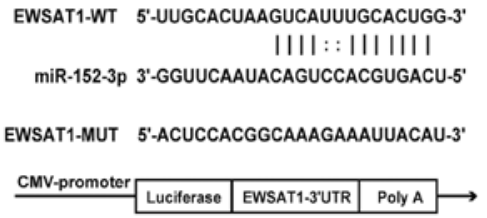

B

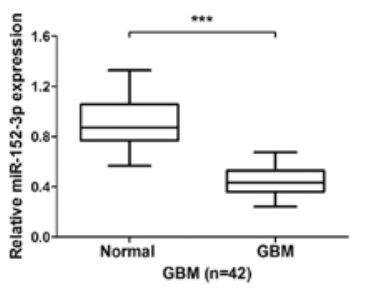

E

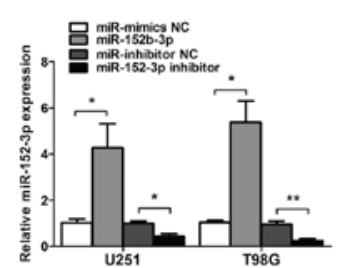

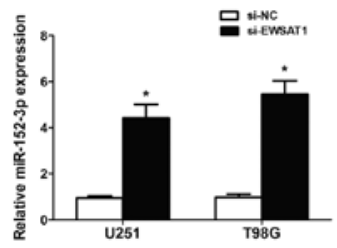

F

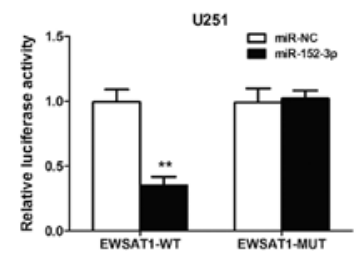

Figure 4. Long non-coding RNA EWSAT1 is targeted by miR-152-3p at the 3'-UTR. (A) Pearson's correlation coefficient analysis between EWSAT1 and miR-152-3p expression levels. (B) The levels of miR-152-3p in GBM compared with adjacent normal tissues were detected using RT-qPCR analysis. ${ }^{* * *} \mathrm{P}<0.001$ (C) miR-152-3p expression was examined by RT-qPCR following transfection with si-EWSAT1 or si-NC in GBM cell lines. (D) Target site of miR-152-3p in the 3'-UTR of EWSAT1. (E) miR-152-3p expression is upregulated by miR-152-3p mimics compared with miR-mimics NC ("P<0.05), or inhibited by miR-152-3p inhibitor compared with the miR-inhibitor $\mathrm{NC}\left({ }^{*} \mathrm{P}<0.05\right.$ and $\left.{ }^{* *} \mathrm{P}<0.01\right)$. (F) The relative luciferase activity was identified following co-transfection of miR-152-3p mimics compared with the miR-NC in EWSAT1-WT- or EWSAT1-MUT-transfected U251 cells using the dual-luciferase reporter assay. ${ }^{* *} \mathrm{P}<0.01$. The experiments were repeated 3 times. EWSAT1, Ewing sarcoma associated transcript 1; miR, microRNA; UTR, untranslated region; GBM, glioblastoma; RT-qPCR, reverse transcription-quantitative PCR; NC, negative control; si, small interfering; WT, wild-type; MUT, mutant.

A

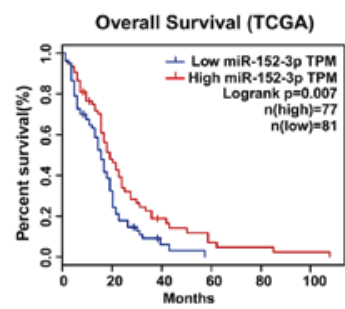

B

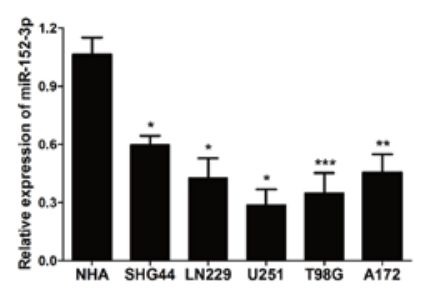

C

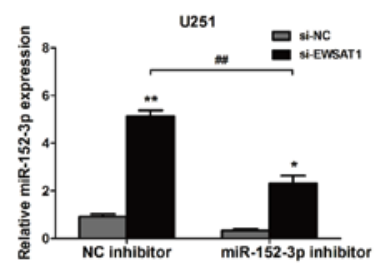

D

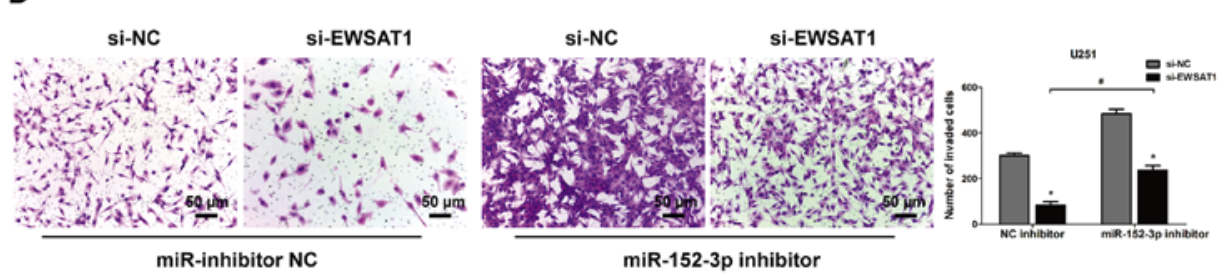

Figure 5. miR-152-3p inhibits the effect of long non-coding RNA EWSAT1 in GBM cells. (A) Kaplan-Meier survival curve analysis of patient data from The Cancer Genome Atlas database with high or low levels of miR-152-3p ( $\mathrm{n}=158)$. (B) miR-152-3p levels in GBM cell lines compared with normal human astrocyte cells were explored by reverse transcription-quantitative PCR. ${ }^{*} \mathrm{P}<0.05,{ }^{* *} \mathrm{P}<0.01$ and ${ }^{* * * *} \mathrm{P}<0.001$ vs. NHA cells. (C) The gene expression levels of miR-152-3p were determined in U251 cells co-transfected with si-EWSAT1 + miR-152-3p inhibitor, and with si-EWSAT1 + miR-inhibitor NC ${ }^{*} \mathrm{P}<0.05$ and ${ }^{* *} \mathrm{P}<0.01$ vs. NC), and in cells co-transfected with si-EWSAT1 + miR-152-3p inhibitor compared with si-EWSAT1 + miR-inhibitor NC (\#\# $\left.<<0.01\right)$. (D) The invasive abilities of the cells were verified using Matrigel assays following transfection with si-EWSAT1 and miR-152-3p inhibitors. Magnification, $\mathrm{x} 100$; scale bar, $50 \mu \mathrm{m} .{ }^{*} \mathrm{P}<0.05$ vs. NC. ${ }^{~} \mathrm{P}<0.05$. The experiment was repeated 3 times. miR, microRNA; EWSAT1, Ewing sarcoma associated transcript 1 ; GBM, glioblastoma; NC, negative control; si, small interfering.

development. To confirm this, the level of EWSAT1 was detected in glioma cell lines (U251, LN229, T98G, A172 and SHG44) and compared with those in NHA cells by RT-qPCR. The results demonstrated that, compared with the NHA cells, the most significant increases in EWSAT1 levels were observed in the U251 and T98G cells (Fig. 2A). Then, an siRNA was used to silence EWSAT1 expression, and significantly decreased expression levels of EWSAT1 were observed compared with those induced by si-NC in both U251 and T98G cells (Fig. 2B).
The result of the CCK- 8 assay revealed inhibited cell viability in EWSAT1-silenced U251 and T98G cells compared with that of cells transfected with the si-NC following 24-72 $\mathrm{h}$ transfection (Fig. 2C). As demonstrated by the results of the Transwell assay, EWSAT1 silencing decreased the number of invasive cells (Fig. 2D). These results revealed that silencing EWSAT1 repressed cell proliferative and invasive abilities in vitro.

To further investigate the underlying mechanism by which EWSAT1 affected the invasive capacity of GBM, TCGA 
database was explored, and a significant positive correlation was identified between EWSAT1 expression and the invasion-associated markers MMP-2 ( $\mathrm{r}=0.26 ; \mathrm{P}=0.00079)$ and MMP-9 ( $\mathrm{r}=0.17 ; \mathrm{P}=0.031)$ (Fig. 3A). Then, the mRNA, protein levels and cellular location of MMP-2 and MMP-9 were determined. Knockdown of EWSAT1 decreased the levels of MMP-2 and MMP-9 in glioma (Fig. 3B and C), and suppressed the expression of the invasion-associated markers MMP-2 and MMP-9 in glioma cells (Fig. 3D). Therefore, these data suggested that EWSAT1 knockdown suppressed both proliferation and invasion in GBM.

Correlation between EWSAT1 and miR-152-3p. StarBase v2.0 (http://starbase.sysu.edu.cn) was employed to predict potential IncRNA-miRNA interactions. A cohort of potential miRNAs that could interact with EWSAT1 was predicted. Pearson's correlation analysis suggested that there was an inverse correlation between EWSAT1 and miR-152-3p level $(n=158 ; r=-0.25$; $\mathrm{P}=0.0011$; Fig. 4A). Then, miR-152-3p expression was detected in 42 GBM and adjacent normal brain samples by RT-qPCR, and a significantly decrease was identified in GBM tissues $(\mathrm{P}<0.001$; Fig. 4B). In addition, the expression of miR-152-3p was significantly upregulated in the EWSAT1-silenced group compared with the si-NC group (Fig. 4C). A luciferase reporter assay was subsequently used to confirm the putative miR-152-3p target site (Fig. 4D). Next, miR-152-3p mimics or inhibitor mimics were used to examine transfection efficiency in the U251 and T98G cell lines (Fig. 4E). In addition, luciferase reporters carrying either the predicted miR-152-3p WT (EWSAT1-WT) or its mutated fragment (EWSAT1-MUT) were constructed. The results from luciferase reporter assays showed that miR-152-3p significantly suppressed the luciferase activity in the cells transfected with the EWSAT1-WT but not mutant EWSAT1-MUT 3'-UTR (Fig. 4F). These data suggested that EWSAT1 may interact with miR-152-3p in glioma cells.

miR-152-3p inhibits the effects of EWSAT1 in GBM cells. The Kaplan-Meier survival analysis demonstrated that low miR-152-3p expression levels were associated with significantly poorer survival in the GEPIA database (Fig. 5A). The level of miR-152-3p in glioma cells was evaluated, and it was identified that miR-152-3p was significantly inhibited in these cells compared with NHA cells (Fig. 5B). Then, rescue experiments were performed using U251 cells. Transfection of the miR-152-3p inhibitor in the si-EWSAT1-transfected group led to a decreased level of miR-152-3p compared with the miR-inhibitor NC (Fig. 5C). The level of miR-152-3p increased when the cells were transfected with si-EWSAT1. However, following treatment with the miR-152-3p inhibitor, the inhibition effect on cell invasion induced by EWSAT1 knockdown was also impeded in the U251 cells (Fig. 5D). These data indicated that miR-152-3p was a vital mediator of EWSAT1-regulated proliferation and invasion processes.

\section{Discussion}

GBM is the most common and aggressive type of central nervous system tumor. Due to its infiltrative growth pattern, anti-apoptotic nature and resistance to chemotherapy, total surgical resection is difficult to perform for this malignant brain tumor. Therefore, it is essential to explore its basic biological mechanisms, and to develop novel targets for recurrence prevention and therapy. It has been previously demonstrated that lncRNAs are involved in a variety of human carcinomas; therefore, an in-depth analysis of these molecules may provide an understanding of the diagnosis, prognosis and therapeutic methods of human cancer (29-32). Moreover, the mechanism by which EWSAT1 serves as an oncogene in GBM requires further study.

In the present study, the level of IncRNA EWSAT1, which is a novel tumor suppressor located on chromosome 15q23, was significantly increased in glioma samples. EWSAT1 overexpression was markedly associated with a poorer prognosis. Moreover, it was demonstrated that EWSAT1 promoted cell proliferation and invasion using gain and loss-of-function assays in vitro. The level of miR-152-3p was decreased in GBM tissues. The expression of miR-152-3p was negatively correlated with that of EWSAT1. By using bioinformatics analysis, miR-152-3p was identified as a promising target of EWSAT1. Together, these results indicated that EWSAT1 exerted a oncogene role in GBM progression, suggesting a potential therapeutic method for GBM.

Previous studies have reported that IncRNAs are potential miRNA sponges $(33,34)$. Silencing of the lncRNA SCAMP1 inhibits malignant biological behavior in glioma through the miR-499a-5p/LIM homeobox transcription factor 1 alpha/NLR family CARD domain containing 5 signaling pathway (35). HOX transcript antisense RNA directly inhibited WIF-1 level by regulating the promoter region methylation of its histone H3K 27 , and then activating the Wnt/ $\beta$-catenin signaling pathway (36). In hepatocellular carcinoma, Zhang et al (37) demonstrated that FLVCR1-AS1 sponges miR-513c to regulate tumor biological functions.

Multiple previous studies have revealed that the abnormal expression of miRNAs serves a vital role in the occurrence and progression of cancer (38). Feng et al (39) showed that miR-152-3p serves as direct regulator of KLF4 in prostate cancer. It was reported that increased EWSAT1 expression was associated with poor outcomes in patients with osteosarcoma (15). Zhang et al (16) suggested that the downregulation of the lncRNA EWSAT1 suppressed cell proliferation and invasion of CRC, which indicated that EWSAT1 may be a potential target of CRC treatment. The lncRNA EWSAT1 promotes ovarian cancer progression through regulating the expression of miR-330-5p (17). Thus, these data appear to indicate an association between the IncRNA EWSAT1 and miR-152-3p in GBM.

In the present study, the putative binding site between EWSAT1 and miR-152-3p was identified using a luciferase reporter assay system; the results confirmed EWSAT1 as a potential tumor suppressor that regulates miR-152-3p, subsequently inhibiting the growth and invasion of glioma. The suppressive effect of si-EWSAT1 was reversed when co-transfected with miR-152-3p inhibitor. Collectively, these results on the role of EWSAT1, and the mechanisms underlying the proliferation and invasion of glioma cells, provide essential information on its role in tumorigenesis.

In conclusion, EWSAT1 acts as an oncogenic lncRNA that facilitates the development and progression of GBM through miR-152-3p, indicating that EWSAT1 may function as a potential biomarker and therapeutic target for GBM. 


\section{Acknowledgements}

Not applicable.

\section{Funding}

The present study was supported by Heilongjiang Health and Health Committee Scientific Research (grant no. 2018377).

\section{Availability of data and materials}

All data generated or analyzed during this study are included in this published article.

\section{Authors' contributions}

HY and WC designed the study, performed experiments, analyzed the data and wrote the manuscript. GJ performed the in vitro experiments. JY and WW analyzed the data and drafted the manuscript. HL designed and supervised the study, and edited the manuscript. All authors read and approved the final manuscript.

\section{Ethics approval and consent to participate}

The present study was approved by the Ethics Committee of The Institutional Review Board of Jiamusi University (Jiamusi, China; approval no. JUIRBR-2019-214) and all procedures were performed in accordance with the principles outlined in The Declaration of Helsinki. Written informed consent was provided by all patients prior to the study start.

\section{Patient consent for publication}

Not applicable.

\section{Competing interests}

The authors declare that they have no competing interests.

\section{References}

1. Lai NS, Wu DG, Fang XG, Lin YC, Chen SS, Li ZB and Xu SS: Serum microRNA-210 as a potential noninvasive biomarker for the diagnosis and prognosis of glioma. Br J Cancer 112: 1241-1246, 2015.

2. Maher EA, Furnari FB, Bachoo RM, Rowitch DH, Louis DN, Cavenee WK and DePinho RA: Malignant glioma: Genetics and biology of a grave matter. Genes Dev 15: 1311-1333, 2001.

3. Davies E, Clarke C and Hopkins A: Malignant cerebral glioma-i: Survival, disability, and morbidity after radiotherapy. BMJ 313 $1507-1512,1996$

4. Sathornsumetee S, Reardon DA, Desjardins A, Quinn JA, Vredenburgh JJ and Rich JN: Molecularly targeted therapy for malignant glioma. Cancer 110: 13-24, 2007.

5. Li C, Jing H, Ma G and Liang P: Allicin induces apoptosis through activation of both intrinsic and extrinsic pathways in glioma cells. Mol Med Rep 17: 5976-5981, 2018.

6. Chen $\mathrm{CC}$, Taniguchi $\mathrm{T}$ and $\mathrm{D}$ 'Andrea A: The Fanconi anemia (FA) pathway confers glioma resistance to DNA alkylating agents. J Mol Med (Berl) 85: 497-509, 2007.

7. Tamura R, Tanaka T, Miyake K, Yoshida K and Sasaki H: Bevacizumab for malignant gliomas: Current indications, mechanisms of action and resistance, and markers of response. Brain Tumor Pathol 34: 62-77, 2017.
8. Li C, Zheng H, Hou W, Bao H, Xiong J, Che W, Gu Y, Sun H and Liang P: Long non-coding RNA linc00645 promotes TGF- $\beta$-induced epithelial-mesenchymal transition by regulating miR-205-3p-ZEB1 axis in glioma. Cell Death Dis 10: 717, 2019.

9. Maruyama R and Suzuki H: Long noncoding RNA involvement in cancer. BMB Rep 45: 604-611, 2012.

10. Spizzo R, Almeida MI, Colombatti A and Calin GA: Long non-coding RNAs and cancer: A new frontier of translational research? Oncogene 31: 4577-4587, 2012.

11. Liu Q, Sun S, Yu W, Jiang J, Zhou F, Qiu G, Xu S and Jiang X: Altered expression of long non-coding RNAs during genotoxic stress-induced cell death in human glioma cells. J Neurooncol 112: 283-292, 2015.

12. Vital AL, Tabernero MD, Castrillo A, Rebelo O, Tão H, Gomes F, Nieto AB, Resende Oliveira C, Lopes MC and Orfao A: Gene expression profiles of human glioblastomas are associated with both tumor cytogenetics and histopathology. Neuro Oncol 12: 991-1003, 2010.

13. Kong L, Li X, Wang H, He G and Tang A: Calycosin inhibits nasopharyngeal carcinoma cells by influencing EWSAT1 expression to regulate the TRAF6-related pathways. Biomed Pharmacother 106: 342-348, 2018.

14. Song P and Yin SC: Long non-coding RNA EWSAT1 promotes human nasopharyngeal carcinoma cell growth in vitro by targeting miR-326/-330-5p. Aging (Albany NY) 8: 2948-2960, 2016.

15. Zhang GY, Zhang JF, Hu XM, Luo ZP and Ma YZ: Clinical significance of long non-coding RNA EWSAT1 as a novel prognostic biomarker in osteosarcoma. Eur Rev Med Pharmacol Sci 21: 5337-5341, 2017.

16. Zhang R, Li JB, Yan XF, Jin K, Li WY, Xu J, Zhao J, Bai JH and Chen YZ: Increased EWSAT1 expression promotes cell proliferation, invasion and epithelial-mesenchymal transition in colorectal cancer. Eur Rev Med Pharmacol Sci 22: 6801-6808, 2018.

17. Fu X, Zhang L, Dan L, Wang K and Xu Y: LncRNA EWSAT1 promotes ovarian cancer progression through targeting miR-330-5p expression. Am J Transl Res 9: 4094-4103, 2017.

18. Marques Howarth M, Simpson D, Ngok SP, Nieves B, Chen R, Siprashvili Z, Vaka D, Breese MR, Crompton BD, Alexe G, et al: Long noncoding RNA EWSAT1-mediated gene repression facilitates Ewing sarcoma oncogenesis. J Clin Invest 124: 5275-5290, 2014.

19. Sempere LF, Freemantle S, Pitha-Rowe I, Moss E, Dmitrovsky E and Ambros V: Expression profiling of mammalian microRNAs uncovers a subset of brain-expressed microRNAs with possible roles in murine and human neuronal differentiation. Genome Biol 5: R13, 2004.

20. Malzkorn B, Wolter M, Liesenberg F, Grzendowski M, Stühler K, Meyer HE and Reifenberger G: Identification and functional characterization of microRNAs involved in the malignant progression of gliomas. Brain Pathol 20: 539-550, 2010.

21. Sun J, Tian X, Zhang J, Huang Y, Lin X, Chen L and Zhang S: Regulation of human glioma cell apoptosis and invasion by miR-152-3p through targeting DNMT1 and regulating NF2: MiR-152-3p regulate glioma cell apoptosis and invasion. J Exp Clin Cancer Res 36: 100, 2017.

22. Ge S, Wang D, Kong Q, Gao W and Sun J: Function of miR-152 as a tumor suppressor in human breast cancer by targeting PIK3CA. Oncol Res 25: 1363-1371, 2017.

23. Liu X, Li J, Qin F and Dai S: MiR-152 as a tumor suppressor microRNA: Target recognition and regulation in cancer. Oncol Lett 11: 3911-3916, 2016.

24. General Assembly of the World Medical Association: World Medical Association Declaration of Helsinki: Ethical principles for medical research involving human subjects. J Am Coll Dent 81: 14-18, 2014.

25. Barrett T, Wilhite SE, Ledoux P, Evangelista C, Kim IF, Tomashevsky M, Marshall KA, Phillippy KH, Sherman PM, Holko M, et al: NCBI GEO: Archive for functional genomics data sets-update. Nucleic Acids Res 41 (Database Issue): D991-D995, 2013.

26. Kong B, Yang T, Chen L, Kuang YQ, Gu JW, Xia X, Cheng L and Zhang JH: Protein-protein interaction network analysis and gene set enrichment analysis in epilepsy patients with brain cancer. J Clin Neurosci 21: 316-319, 2014.

27. Livak KJ and Schmittgen TD: Analysis of relative gene expression data using real-time quantitative PCR and the 2(-Delta Delta C(T)) method. Methods 25: 402-408, 2001. 
28. Benjamini Y and Hochberg Y: Controlling the false discovery rate: A practical and powerful approach to multiple testing. J R Stat Soc B 57: 289-300, 1995.

29. Xu T, Lin CM, Cheng SQ, Min J, Li L, Meng XM, Huang C, Zhang L, Deng ZY and Li J: Pathological bases and clinical impact of long noncoding RNAs in prostate cancer: A new budding star. Mol Cancer 17: 103, 2018.

30. Lalevee S and Feil R. Long noncoding RNAs in human disease: Emerging mechanisms and therapeutic strategies. Epigenomics 7: 877-879, 2015.

31. Bhan A and Mandal SS: Long noncoding RNAs: Emerging stars in gene regulation, epigenetics and human disease. ChemMedChem 9: 1932-1956, 2014.

32. Yarmishyn AA and Kurochkin IV: Long noncoding RNAs: A potential novel class of cancer biomarkers. Front Genet 6: 145 2015.

33. Hu Y, Deng C, Zhang H, Zhang J, Peng B and Hu C: Long non-coding RNA XIST promotes cell growth and metastasis through regulating miR-139-5p mediated Wnt $/ \beta$-catenin signaling pathway in bladder cancer. Oncotarget 8: 94554-94568, 2017.

34. Li C, Wan L, Liu Z, Xu G, Wang S, Su Z, Zhang Y, Zhang C, Liu X, Lei Z and Zhang HT: Long non-coding RNA XIST promotes TGF- $\beta$-induced epithelial-mesenchymal transition by regulating miR-367/141-ZEB2 axis in non-small cell lung cancer Cancer Lett 418: 185-195, 2018.
35. Zong Z, Song Y, Xue Y, Ruan X, Liu X, Yang C, Zheng J, Cao S, Li Z and Liu Y: Knockdown of LncRNA SCAMP1 suppressed malignant biological behaviours of glioma cells via modulating miR-499a-5p/LMX1A/NLRC5 pathway. J Cell Mol Med 23: 5048-5062, 2019

36. Ge XS, Ma HJ, Zheng XH, Ruan HL, Liao XY, Xue WQ, Chen YB, Zhang Y and Jia WH: HOTAIR, a prognostic factor in esophageal squamous cell carcinoma, inhibits WIF-1 expression and activates Wnt pathway. Cancer Sci 104: 1675-1682, 2013.

37. Zhang K, Zhao Z, Yu J, Chen W, Xu Q and Chen L: LncRNA FLVCR1-AS1 acts as miR-513c sponge to modulate cancer cell proliferation, migration, and invasion in hepatocellular carcinoma. J Cell Biochem 119: 6045-6056, 2018.

38. Calin GA and Croce CM: MicroRNA signatures in human cancers. Nat Rev Cancer 6: 857-866, 2006.

39. Feng F, Liu H, Chen A, Xia Q, Zhao Y, Jin X and Huang J: miR-148-3p and miR-152-3p synergistically regulate prostate cancer progression via repressing KLF4. J Cell Biochem 120: 17228-17239, 2019.

This work is licensed under a Creative Commons Attribution-NonCommercial-NoDerivatives 4.0 International (CC BY-NC-ND 4.0) License. 